\title{
MACRO-ECONOMETRIC MODELLING FOR THE NIGERIAN ECONOMY: GROWTH-POVERTY GAP ANALYSIS
}

By

\section{OLUSEGUN AYODELE AKANBI}

Submitted in partial fulfillment of the requirements for the degree DOCTOR OF PHILOSOPHY (ECONOMICS)

in the

Faculty of Economics and Management Sciences

at the

UNIVERSITY OF PRETORIA 


\title{
MACROECONOMETRIC MODELLING FOR THE NIGERIAN ECONOMY: A GROWTH-POVERTY GAP ANALYSIS
}

By

\section{OLUSEGUN AYODELE AKANBI}

SUPERVISOR

CO-SUPERVISOR

DEPARTMENT

DEGREE
PROF. CHARLOTTE B. DU TOIT

PROF. RANGAN GUPTA

ECONOMICS

DOCTOR OF PHILOSOPHY (ECONOMICS)

\begin{abstract}
This study develops comprehensive full-sector macro-econometric models for the Nigerian economy with the aim of explaining and providing a long-term solution for the persistent growth-poverty divergence experienced by the country. The models are applied to testing the hypothesis of existing structural supply-side constraints versus demand-side constraints impeding the growth and development of the country.
\end{abstract}

Structural inadequacies have been the major setback to the achievement of the developmental objectives in the Nigerian economy. The last four decades has revealed several macroeconomic instabilities that hinder much improvement in the performance of the economy. Much of these structural inadequacies have been blamed on the persistent poor governance experienced by the country over the years. The poor political leadership and instability, corruption and the mismanagement of the oil resources have halted an appropriate and sound economic policy that should have alleviated poverty among the majority of the population. 
A review of the historical performance of the Nigerian economy reveals significant socio-economic constraints as the predominant impediments to high and sticky levels of poverty in the economy. As such, a model of the Nigerian economy suitable for policy analysis needs to capture the long-run supply-side characteristics of the economy. A price block is incorporated to specify the price adjustment between the production or supply-side sector and real aggregate demand sector. The institutional characteristics with associated policy behaviour are incorporated through a public and monetary sector, whereas the interaction with the rest of the world is presented by a foreign sector, with specific attention given to the oil sector.

The models are estimated with time-series data from 1970 to 2006 using the EngleGranger two-step cointegration technique, capturing both the long-run and short-run dynamic properties of the economy. The full-sector models are subjected to a series of policy scenarios to evaluate the various options for government.

It is evident from the policy options assessed in this study that there is a need for an improvement in the quality of government spending. Fiscal policy expansion should tend towards increasing the component of government expenditure that will lead to sustained growth and also an improvement in the standard of living of the citizens. In order to be able to reap the benefits of a positive external shock, there is a need to increase the level of competitiveness and the productive capacity of the country. Investment in basic infrastructure such as power and roads is very crucial at this stage of the Nigerian economy.

There is an urgent need to refocus the government role in certain critical areas of the economy. Government institutions need to be strengthened by improving the coordination that exists within the government structures. The political environment needs to be more secure in order to attract more private investment. The maintenance of public order, ensuring property rights, a sound regulatory structure and also creating a framework that will increase the provision of public goods and services and the maintenance of infrastructure are urgent elements required in order to achieve the set macroeconomic objectives. 


\section{TABLE OF CONTENTS}

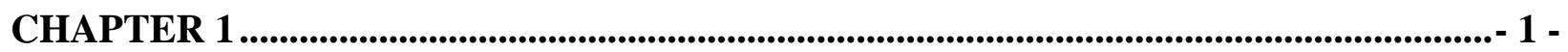

BACKGROUND AND INTRODUCTION............................................................................... 1 -

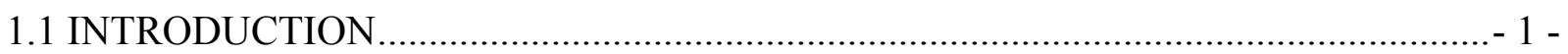

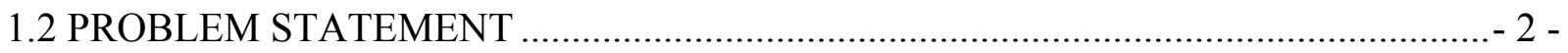

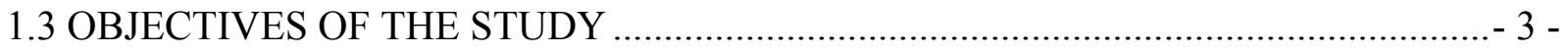

1.4 RESEARCH METHODOLOGY .................................................................................. 3 -

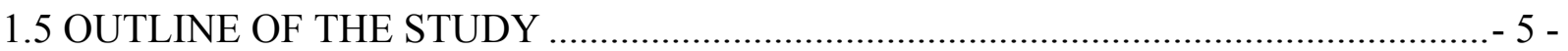

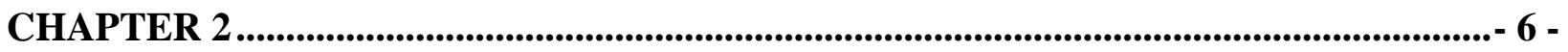

THEORETICAL ANALYSIS: GROWTH AND POVERTY .............................................. 6 -

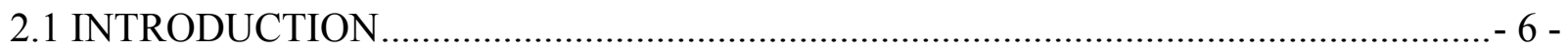

2.2 THEORETICAL GROWTH MODEL ANALYSIS ......................................................... 6 -

2.3 THEORETICAL PRO-POOR GROWTH (POVERTY TRAP) MODEL ANALYSIS...- 8 -

2.4 GROWTH AND POVERTY EMPIRICS ................................................................... 14 -

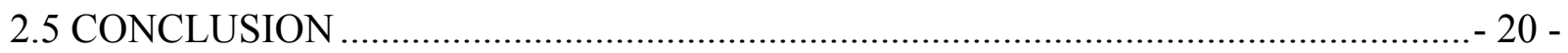

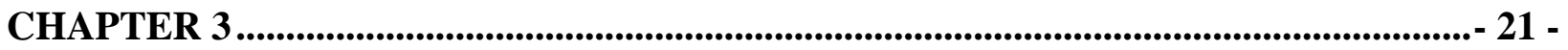

EVALUATING THE GROWTH AND POVERTY PERFORMANCE OF NIGERIA ..- 21 -

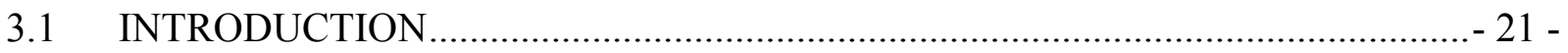

3.2 NIGERIAN ECONOMIC GROWTH PERFORMANCE ...............................................- 21 -

3.2.1 Wealth of the Nigerian economy .......................................................................... 21 -

3.2.2 The evolution of the Nigerian economy ………................................................... 26 -

3.2.3 Sources of Economic Growth (Growth Accounting) .................................................- 34 -

3.3 NIGERIA POVERTY PERFORMANCE …………….............................................. 38 - 
3.3.1 Measurement of Poverty ……………………….................................................... 38 -

3.3.2 Profile of poverty in Nigeria.................................................................................... 39 -

3.3.3 Poverty reduction strategies in Nigeria .................................................................. 44 -

3.2.4: Challenges to poverty alleviation in Nigeria …….................................................. 47 -

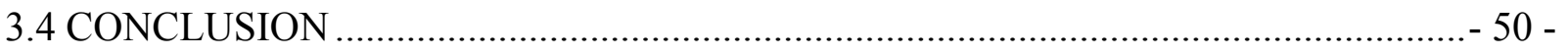

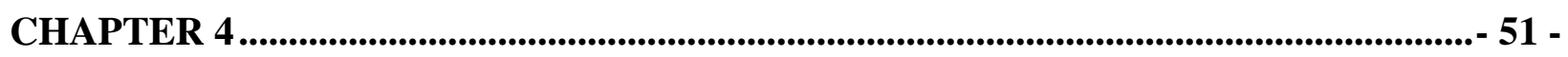
MODEL SPECIFICATION AND ESTIMATION TECHNIQUES ..................................... 51 -

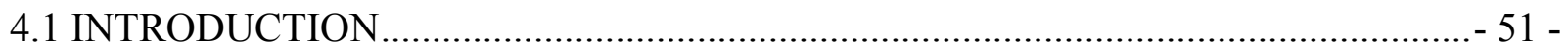

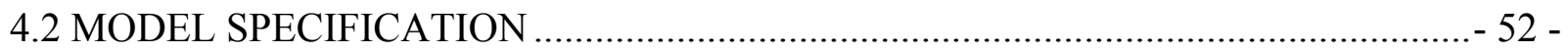

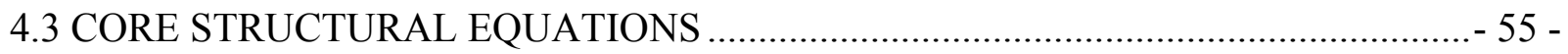

4.3.1 The real sector …………………................................................................... 55 -

4.3.2 The external sector............................................................................................. 63 -

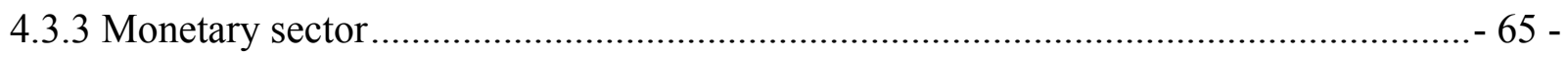

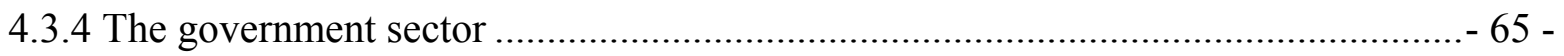

4.3.5 Other behavioural equations in the model .................................................................- 66 -

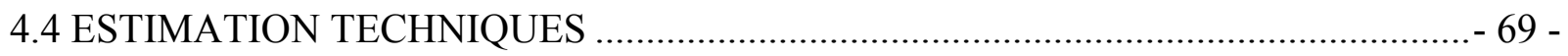

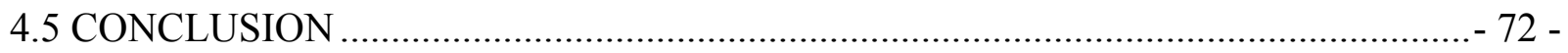

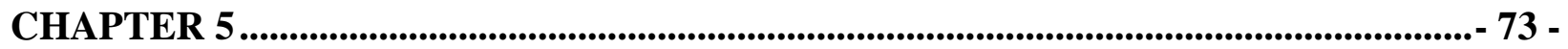

EMPIRICAL ANALYSIS ................................................................................................................ 73 -

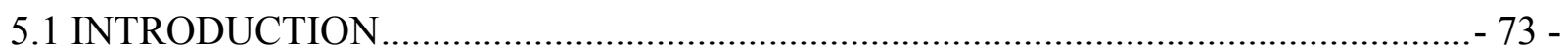

5.2 ESTIMATION RESULTS (MODEL A): SUPPLY-SIDE ORIENTATED ...................... 74 -

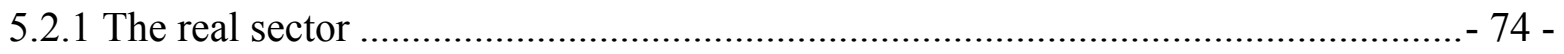

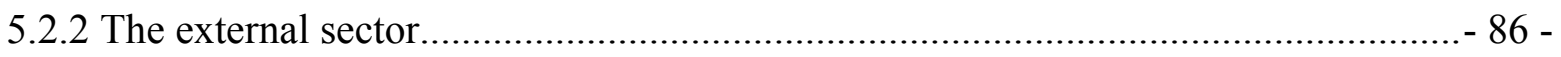

5.2.3 The monetary sector ……………..................................................................... 90 - 
5.2.4 Other behavioural equations in the model. $-91-$

5.3 ESTIMATION RESULTS (MODEL B): DEMAND-SIDE ORIENTATED .. - $96-$

5.4 MODEL CLOSURES - 101 -

5.6 CONCLUSION $-113-$

CHAPTER 6. $114-$ SUMMARY, CONCLUSION AND POLICY RECOMMENDATIONS ...........................- 114 -

6.1 INTRODUCTION. -114 -

6.2 SUMMARY AND MAIN FINDINGS OF THE STUDY $-114-$

6.3 POLICY RECOMMENDATIONS. -117 6.4 AREAS FOR FURTHER RESEARCH. $-119-$ 6.5 FINAL CONCLUSION - $119-$

REFERENCES................................................................................................................................ 121 -

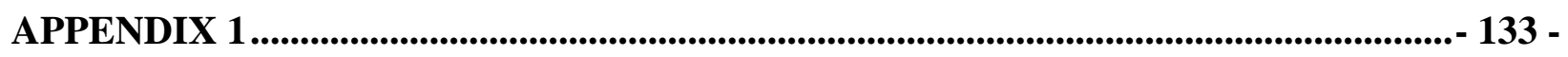
AN EXPOSITION OF THE DATA UTILISED IN THE MODEL ..................................... 133 -

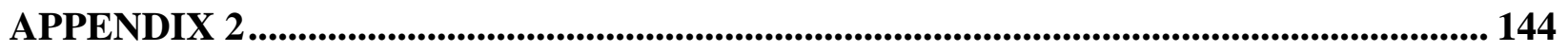

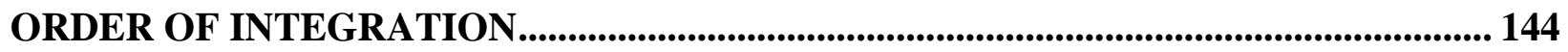
APPENDIX 3 ............................................................................................................................................... 152

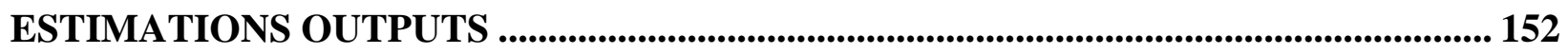

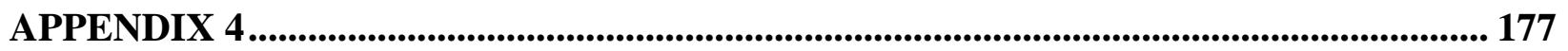

MODEL SIMULATIONS: ACTUAL AND FITTED VALUES............................................ 177

APPENDIX 5 .................................................................................................................................. 184

EQUATIONS, IDENTITIES, AND ESTIMATED PARAMETERS OF THE FULL

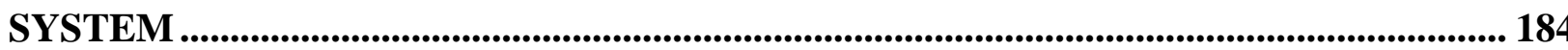




\section{LIST OF TABLES}

Table 2.1: Summary of Empirical Literature Related to Pro-Poor Growth Model $14-$

Table 3.1: Nigeria Selected Petroleum Statistics, 2000-2006 (Millions; Barrels) $-22-$

Table 3.2: Total Exports of selected African countries, 2006 (values in billions of current US\$)..- 22 -

Table 3.3: Major Economic Indicators. $-29-$

Table 3.4: Economic Performance 1999-2005. $-32-$

Table 3.5: Sectoral Growth Rate $-33-$

Table 3.6: Sources of Economic Growth in Nigeria (Per cent per Year). $-36-$

Table 3.7: Nigerian Human Development and Poverty Index. $39-$

Table 3.8: Nigerian Poverty Profile 1980/1996 (\% Population) $-40-$

Table A1: Data Description and Calculation 137

Table A2.1: Augmented Dickey-Fuller tests for non-stationarity, levels, 1970-2006. 146

Table A2.2: Augmented Dickey-Fuller tests for non-stationarity, first differences, 1970-2006 148

Table A3.1: Model A 152

Table A3.2: Model B. 166 


\section{LIST OF FIGURES}

Figure 3.1 Nigeria Trade Account (1970-2006).......................................................................- 23 -

Figure 3.2: Divergence of Oil and Non-Oil Exports in Nigeria....................................................- 24 -

Figure 3.3 Oil Production, Total Exports, Total Imports as a Share of GDP ……..........................- 25 -

Figure 3.4 Total Value of Oil Production in Nigeria (1970-2006) .................................................- 27 -

Figure 3.5 Real per capita GDP and Household Consumption Expenditure (1970-2006) ...............- 27 -

Figure 3.6 Exchange Rate and Inflation Rate 1970-2006 .............................................................- 31 -

Figure 3.7: Nigeria Inequality Relative to other Countries .........................................................- 48 -

Figure 3.8: Nigeria's Economic Growth-Poverty Performance ......................................................... 49 -

Figure 4.1: Demand-side Fuelled Growth Strategy ......................................................................... 53 -

Figure 5.1: A Flow Chart of the Model..................................................................................... 103 -

Figure 5.2A: Shock on Total Government Expenditure (Model A) ………………………........... 105 -

Figure 5.2B: Shock on Total Government Expenditure (Model B).............................................- 106 -

Figure 5.3A: Shock on World Oil Prices (Model A) ………....................................................... 107 -

Figure 5.3B: Shock on World Oil Prices (Model B) ...................................................................- 108 -

Figure 5.4A: Shock on World Income (Model A) …………...................................................... 110 -

Figure 5.4B: Shock on World Income (Model B)....................................................................- 110 -

Figure 5.5A: Shock on Government Effectiveness; Worse Governance (Model A) ......................- 112 -

Figure 5.5B: Shock on Government Effectiveness; Worse Governance (Model B) ...................... 112 - 
Figure A3.1: Long-Run Residuals (Model A) 171

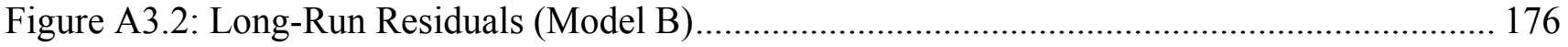

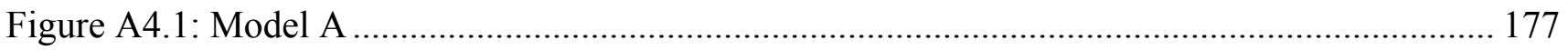

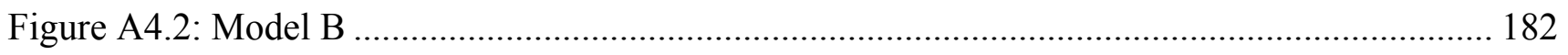

